\title{
A Typical Neurological Presentations in the ICU: Limbic Encephalitis
}

\author{
Purvesh R. Patel, Rubin I. Cohen and Seth Koenig*
}

\author{
The Division of Pulmonary, Critical Care and Sleep Medicine, The North Shore-Long Island Jewish Health System, The \\ Hofstra North Shore-LIJ School of Medicine, New Hyde Park, NY, USA
}

\begin{abstract}
Common neurological emergencies include overdose or withdrawal from illegal substance abuse, adverse effects of prescription medications, seizures, metabolic encephalopathy, infections and cerebrovascular accidents. Following a thorough clinical and radiologic assessment, a small group of patients escape definitive diagnosis and autoimmune encephalitides should be considered. Of these, limbic encephalitis (LE) is the most common and may result from paraneoplastic or nonparaneoplastic sources. Common to both is the production of antibodies targeting epitopes in the brain parenchyma thought to be responsible for the clinical manifestations. Paraneoplastic Anti-N-methyl D-aspartate receptor (NMDAR) encephalitis is a common cause of LE and has gained awareness in neurological and psychiatric literature. Paraneoplastic and nonparaneoplastic anti NMDAR encephalitis typically presents in young, previously healthy females with subacute onset of psychiatric symptoms, respiratory insufficiency, orofacial dyskinesias, autonomic instability and seizures. Paraneoplastic LE is induced by antibody production against NMDAR with occult ovarian teratoma being the most common inciting tumor. LE has also been described in association with other tumors and also without tumors. The latter are known as nonparaneoplastic or primary autoimmune disease. Diagnosis requires both clinical suspicion along with prompt serum and cerebrospinal fluid analysis for antibody detection. Immunotherapy to remove and suppress these antibodies along with resection of an identified tumor is the therapy of choice. This article will review the clinical presentation and management of LE in patients who present to the medical intensive care unit.
\end{abstract}

Keywords: Limbic encephalitis, paraneoplastic, encephalitis, autoimmune encephalitis.

\section{INTRODUCTION}

Encephalitis syndromes are common medical emergencies encountered by critical care physicians. These patients require early diagnosis and appropriate treatment as delay in could lead to irreversible brain injury or death. Common causes include toxic (overdose, withdrawal and adverse effects of prescription medications), seizures, metabolic encephalopathy, infections (meningitis and/or encephalitis) and cerebrovascular accidents. After thorough clinical and radiologic examinations, a small group of patients escape definitive diagnosis. Once systemic autoimmune diseases and thyroid autoimmunity have been ruled out; autoimmune encephalitides must be considered. Most common of all autoimmune encephalitides is Limbic Encephalitis (LE) and is the focus of this review. Despite the growing recognition of autoimmune encephalitis, this entity remains poorly understood and diagnosis is often considered late and often not at all, resulting in poor outcome. Common presentations of LE to the critical care unit include respiratory failure, seizure and movement disorders.

A large review of over 1500 patients presenting with encephalitis to health care facilities in California showed that only $16 \%$ of cases had a confirmed etiological agent, $13 \%$ had a suspected etiological agent and $8 \%$ had a noninfectious

*Address correspondence to this author at the Division of Pulmonary, Critical Care and Sleep Medicine, The North Shore-Long Island Jewish Health System, The Hofstra North Shore-LIJ School of Medicine, New Hyde Park, NY, USA; Tel: 01 516-465-5400; Fax: 01516465 5454;

E-mail: Skoenig@nshs.edu cause with autoimmune disease and vasculitides being the most common. An etiology was not found in nearly two thirds of cases referred to specialized units $[1,2]$. This highlights the importance of considering autoimmune encephalitis earlier where no clear infectious or inflammatory etiology is identified.

The intention of this article is to raise awareness of autoimmune encephalitides, a potentially reversible cause of a common medical emergency.

The Limbic system is formed of the amygdala, hippocampus and hypothalamus and is responsible for memory, emotion and behavior. LE is either a paraneoplastic or nonparaneoplastic syndrome. Common tumors causing paraneoplastic LE are teratomas, small cell lung cancer (SCLC), breast cancer, testicular tumors, Hodgkin's lymphoma and thymomas [3]. Neurological symptoms may precede the diagnosis of malignancy in up to $60 \%-75 \%$ of such patients [3,4]. Nonparaneoplastic LE occurs in the absence of malignancy, and is termed primary or idiopathic LE $[5,6]$. Of all causes of LE, paraneoplastic anti-NMDAR encephalitis is more common and treatable, with an excellent prognosis if recognized and treated early. The incidence of paraneoplastic anti-NMDAR encephalitis is not known but likely is under-recognized [7]. In one series, $1 \%$ of those aged 18-35 admitted to ICU with encephalitis of unknown cause were seropositive for NMDA receptor antibody [8]. Paraneoplastic anti NMDAR encephalitis typically manifests in young female patients who harbor an ovarian teratoma; however other malignancies have also been implicated [8]. 


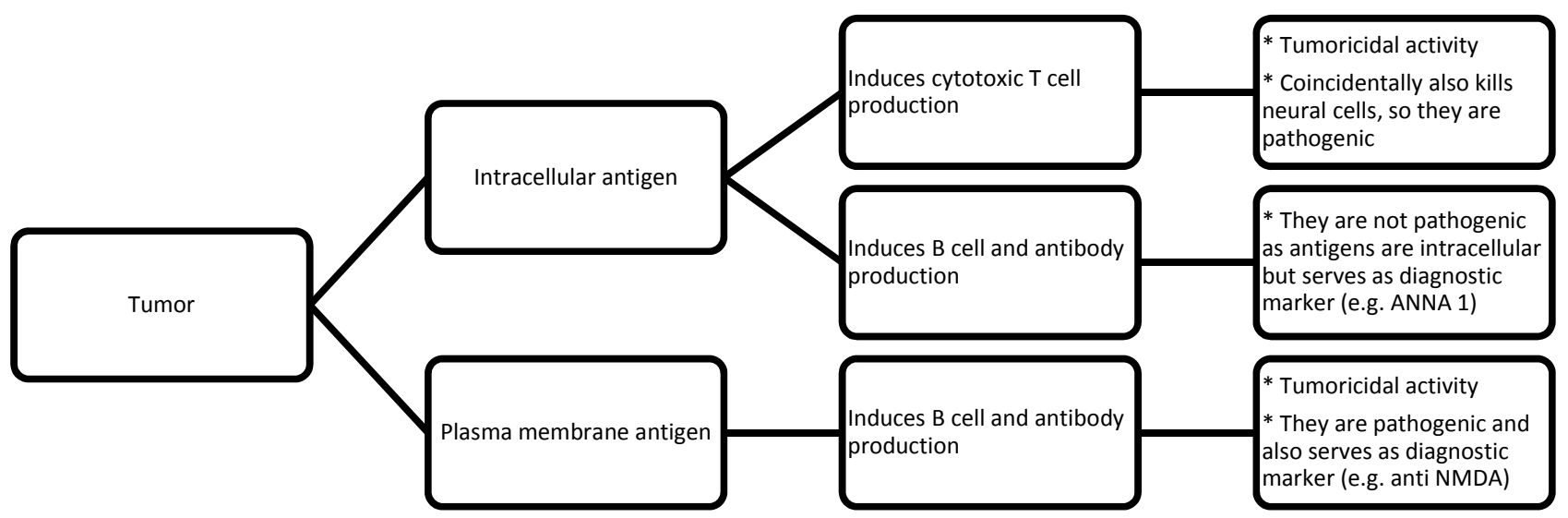

Fig. (1). Paraneoplastic immunopathogenic mechanisms. Tumor initiates immune response against onconeural proteins. Tumor antigens are presented to adaptive immune system, which results in activation of immune system. These antigens are also normally expressed in neural cells and are thus coincidental targets. Intracellular antigens induce cytotoxic T cells (CD8+) and are effectors of immune mediated cellular injury. They also induce antibody production (e.g. PCA 1 or anti Yo). These antibodies are not pathogenic but serve as diagnostic markers of a $\mathrm{T}$ cell predominant effector process. Plasma membrane antigens induce antibody production (e.g. anti NMDAR). These antibodies are pathogenic and are effectors of immune mediated cellular dysfunction and injury.

\section{HISTORY}

In 1960, Brierley and colleagues reported three patients as "subacute encephalitis of later adult life, mainly affecting the limbic areas". Corsellis and associates used the word "limbic encephalitis" and established a relationship between limbic encephalitis and systemic cancer. He described patients with severe short term memory impairment or dementia in association with bronchial carcinoma [9]. For the next 20 years, LE was considered a rare disorder that almost always occurred in association with cancer. Following advances in neuroimaging and the discovery of paraneoplastic antibodies, recognition of this disorder increased, suggesting that its frequency had been underestimated. During this time several clinical immunological associations were established. These include SCLC and anti-Hu, germ cell tumor of testis and anti-Ma2 antibody. Increased awareness of the clinical manifestations of LE led to the identification of patients with similar syndromes but without paraneoplastic antibodies or tumors.

\section{PATHOGENESIS}

The immune system dysfunction results in brain pathology and some of these mechanisms have been well studied in systemic lupus erythematosus neuro-vasculitis and thrombosis and in multiple sclerosis. In addition many more individuals with functional brain disorders or structural lesions once thought to be non-immune related now have their symptoms attributed to the immune response. Immunemediated damage to the CNS might occur more commonly than once recognized [10].

The role of antibodies in the pathogenesis of autoimmune encephalitides is crucial. Numerous brain reactive antibodies have been identified in the serum and may be associated with neurological and neuropsychiatric symptoms. Some of these antibodies are directly responsible for immune mediated damage; whereas others are mere markers for the presence of the disease.

Antineuronal antibodies are generally classified according to the location of the recognized antigen into two groups: 1. Intraneuronal antigens, and 2. Cell membrane antigens. Neurological disorders associated with these antibodies can be linked either to an underlying tumor (paraneoplastic neurological syndrome) or have an unknown origin as primary autoimmune response against the nervous system (idiopathic or nonparaneoplastic neurological syndrome).

Paraneoplastic neurological disorders in which the target antigens are intracellular are caused by CD8+ cytotoxic T cells mediated autoimmunity. In this case, the antibodies targeting these intracellular antigens are used for diagnostic purposes only [11] (Fig. 1). Prototypic example is cerebellar degeneration occurring in patients who are seropositive for Purkinje cell cytoplasmic antibody type 1 (PCA 1 or anti Yo). This autoantibody predicts with $90 \%$ certainty the presence of adenocarcinoma of the ovary, uterus, fallopian tube, peritoneum or breast [11]. The antigen is intracellular and inaccessible in intact cells to circulating antibody. Thus, these antibodies are not pathogenic and the neurological deficit rarely improves with antibody-depleting or tumoricidal therapies.

Paraneoplastic neurological disorders in which the target antigens are present on the plasma membrane are caused by antibody-mediated autoimmunity. Antibodies targeting the neural cell surface antigens such as receptors and ion channels have a pathogenic role in paraneoplastic disorders affecting the nervous system. A prototypic example is encephalopathy secondary to antibodies targeting the ionotropic glutamate receptor also known as NR1 subunit of N-methyl D-aspartate - NMDA receptor. This autoantibody is often associated with ovarian teratoma. Because these antibodies are pathogenic, symptoms generally improve with early teratoma removal and immunotherapy targeted at both removing antibodies using plasmapheresis or intravenous immunoglobulin (IVIG) and reducing their production with steroids and chemotherapy. This emphasizes the need for prompt diagnosis and initiation of treatment in critically ill patients presenting with signs and symptoms suggestive of LE. 


\section{CLINICAL PRESENTATION}

Autoimmune encephalitides are a spectrum of disorders involving central, peripheral somatic or peripheral autonomic nervous systems. Classical syndromes described are LE, brainstem encephalitis, encephalomyelitis, opsoclonusmyoclonus and cerebellar degeneration. However they often have multifocal presentations. Common antibodies and their associated antigens, neoplasm and phenotype are outlined in the Tables $\mathbf{1}$ and $\mathbf{2}$.

Paraneoplastic anti-NMDAR LE frequently affects young women between the second and fifth decade of life. Patients initially have prominent psychiatric manifestations and patients are frequently admitted to a psychiatric ward. Symptoms include anxiety, depression, irritability, hallucinations and personality change. Short term memory deficit is the hallmark of this disorder. Movement disorders are also very common and most patients develop dystonic or catatonic posture, symmetrical limb movements, and typical orofacial dyskinesias. Most patients have an altered level of consciousness ranging from mere confusion to obtundation. In addition, most patients develop seizures (complex partial, secondary generalized seizures) requiring anti-epileptic drugs (AEDs). They also develop autonomic instability including fluctuations in temperature, heart rate and blood pressure along with profuse sweating, and excessive salivation $[12,13]$. Severe autonomic manifestations have been described such as hyerpyrexia, cardiac pauses and tachycardia-bradycardia syndromes.

It has been suggested that an infectious prodromal headache and fever is followed by a cortical stage and subcortical stage of disease with a 10 to 20 day interval between the two stages [14]. The Cortical stage is characterized by neuropsychiatric symptoms, seizures, CSF lymphocytosis in the absence of oligoclonal bands, epileptiform discharges on EEG and cortical MRI changes. The Subcortical stage of disease is characterized by movement disorders, dysautonomia, a decreased level of consciousness and CSF oligoclonal bands in the absence of lymphocytosis and diffuse slowing on EEG and sub-cortical MRI changes [14]. These phases may correlate well with NMDAR antibodies altering the function of the cortical neurons, in conjunction with CNS lymphocyte influx, to produce downstream disinhibition of central pattern generators resulting in the development of movement disorders [15]. The unique movement disorder consist of repetitive semirhythmic ocular, jaw, facial, lingual, limb and trunk movements. Examples are mouth opening and closing, bruxism, chewing, facial grimacing, frowning, lip pouting, tongue protrusion, oculogyric deviation, undulating to jerky limb movements, dystonic limb posturing and opisthotonus [15]. Movement disorder is influenced by sensory stimulation even though they occur during depressed consciousness. The movement disorders resolve as responsiveness returns. The correlation between clinical course and antibody titers suggest the NMDAR antibodies are pathogenic.

ICU admission is often required for management of seizures with AEDs, decreased level of consciousness, prominent hyperkinetic movement disorder of limbs, trunk and orofacial muscles with tongue and teeth injury and autonomic instability. Patients often require sedation and frequently mechanical ventilation. After controlling seizures, attempts to decrease sedation shows limited recovery of consciousness and spontaneous breathing with recurrence or worsening of abnormal movements and weaning may be prolonged. In our experience and that of others, tracheostomy and percutaneous endoscopic gastrostomy tubes were required in most patients.

Data from a Japanese survey of paraneoplastic antiNMDAR encephalitis [16], indicated fever and psychosis were the presenting symptoms in $90 \%$. Among the neurological symptoms, disturbance of consciousness was present in $92 \%$, convulsions in $65 \%$, and involuntary movements in $55 \%$. Respiratory failure on admission was observed in $30 \%$ of patients, but mechanical ventilation was eventually required in $78 \%$ of patients. Among the patients who required mechanical ventilation, $91 \%$ suffered central respiratory failure without respiratory infection and the

Table 1. Antibodies Targeting Intracellular Antigens

\begin{tabular}{|c|c|c|c|}
\hline Antibody & Antigen & Neoplasm & Phenotype \\
\hline \hline ANNA-1 & ELAVL (Hu) & SCLC & Neuropathies (autonomic, peripheral and cranial), LE \\
\hline ANNA-2 & NOVA 1,2 (Ri) & SCLC, Breast adenocarcinoma & LE, Brainstem encephalitis (jaw dystonia, laryngospasm), Cerebellar ataxia \\
\hline PCA-1 & CDR2 & $\begin{array}{c}\text { Mullerian adenocarcinoma, Breast } \\
\text { adenocarcinoma }\end{array}$ & Cerebellar ataxia, Brainstem encephalitis, Myelopathy, Peripheral neuropathy \\
\hline
\end{tabular}

ANNA: antineuronal antibody, ELAVL: embryonic lethal abnormal vision like, NOVA: Neuro-oncologic ventral antigen, PCA: Purkinje cell antibody, CDR: cerebellar degeneration related protein.

Table 2. Antibodies Targeting Cell Membrane Antigens

\begin{tabular}{|c|c|c|c|}
\hline Antibody & Antigen & Neoplasm & Phenotype \\
\hline \hline VGKC complex antibody & LGI1, CASPR2 & $\begin{array}{c}\text { SCLC, Thymoma, Breast and prostate } \\
\text { adenocarcinoma }\end{array}$ & $\begin{array}{c}\text { LE, Amnestic syndrome, Personality change, } \\
\text { Brainstem encephalitis, Peripheral neuropathy }\end{array}$ \\
\hline NMDA receptor antibody & NR1 & Ovarian teratoma & LE, Psychosis, Extrapyramidal disorders \\
\hline AMPA receptor antibody & GluR1, 2 & Thymic tumor, Lung carcinoma, Breast carcinoma & LE, Nystagmus, Seizures \\
\hline
\end{tabular}

VGKC: voltage gated potassium channel, LGI1: leucine rich glioma inactivated 1, CASPR2: contactin associated protein like 2, NMDA: N-methyl-D-aspartate, AMPA: $\alpha$-amino-3hydroxy-5-methyl-4-isoxazolepropionic acid. 
remaining $9 \%$ received mechanical ventilation because of general anesthesia for seizures control. The duration of care with mechanical ventilation was $102 \pm 153$ days (mean and SD) with a maximum of 933 days. In another case series the median duration of ventilatory support was 12 weeks [7].

\section{DIAGNOSIS}

\section{General Considerations}

Autoimmune encephalitis is a diagnosis of exclusion. Initial investigation in an encephalitic patient would include computed tomography of the brain, lumbar puncture for CSF analysis of cell count, protein, glucose, bacterial, mycobacterial and viral culture. MRI and EEG should be considered depending on the clinical presentation.

HSV encephalitis is promptly ruled out by HSV PCR with very good accuracy, although this may be negative when sampled within the first 72 hours of symptom onset. Other viral encephalitides such as varicella zoster virus and cytomegalo virus must also be considered in the differential diagnosis. Human herpes virus 6 is identical in presentation to LE and should be excluded in immunosuppressed patients.

Following the elimination of infectious etiologies, systemic autoimmune disorders such as SLE, Sjogren's syndrome and antiphospholipid syndrome should also be excluded (Table 3).

Patients should be evaluated for thyroid autoimmunity or Hashimoto's thyroiditis with serum thyroperoxidase or thyroglobulin antibodies. These diseases may present with subacute cognitive disorder with tremor, myoclonus, neuropsychiatric symptoms and seizures similar to autoimmune encephalitides and often without clinical or biochemical evidence of thyroid dysfunction. They typically improve with corticosteroid therapy but may relapse [17-20].

\section{Specific Considerations}

Radiology: Differentiating paraneoplastic from nonparaneoplastic LE may be difficult as $70 \%$ of paraneoplastic cases the neurologic disorder precedes the detection of the tumor. Conversely similar clinical, CSF and radiologic findings may occur without cancer association. Accordingly patient should undergo a thorough physical examination along with CT chest, abdomen and pelvis \&/or PET-CT (FDG) for the detection of subclinical malignancy. PET-CT may also demonstrate CNS abnormalities.

In anti NMDAR encephalitis CT scans are usually noncontributory. The MRI is either unremarkable or atypical in up to $75 \%$ of patients. In the other $25 \%$, the MRI shows typical T2 or FLAIR (fluid attenuated inversion recovery) signal hyperintensity in the medial temporal lobes [7]. Atypical MRI findings include mild, transient T2 or FLAIR abnormalities outside the medial temporal lobes, sometimes with cortical enhancement. PET-CT (FDG) is helpful in tumor detection and has also been demonstrated to show frontotemporal to occipital gradient in anti NMDAR encephalitis [21].

CSF findings: CSF findings are nonspecific. The most common CSF abnormalities are lymphocytic pleocytosis, elevated protein with normal glucose and oligoclonal bands suggesting autoantibody presence. CSF should be tested for anti-neuronal autoantibody analysis.

EEG findings: Most patients with anti NMDAR encephalitis develop generalized or partial complex seizure [9]. EEG findings show diffuse slowing and/or paroxysmal wave in $89 \%$ of patients [16].

Serology: Accurate detection of anti-neuronal antibodies has important implications for the clinical investigation and treatment of patients with autoimmune encephalitis. However, seronegativity does not exclude a paraneoplastic etiology [11]. A battery of autoimmune antibody panels is available commercially; however interpretation of results requires experience and must be put in the context of clinical information. False negative and positives on antibody screening may further complicate the clinical picture.

\section{TREATMENT}

The mechanisms of neuronal dysfunction in autoimmune encephalitides (paraneoplastic or nonparaneoplastic) are not well understood. However, clinical experience, neuropathologic studies, and response to empiric treatment suggest two immune mediated mechanisms, depending on the location of the antigen. As noted in the pathogenesis section, 1. The encephalitides related to intracellular antigens, predominantly caused by cytotoxic $\mathrm{T}$ cell effectors and 2. The encephalitides related to cell surface antigens, predominantly caused by antibody effectors.

The first group of encephalitides are paraneoplastic, associated with certain cancers. Usually, brain infiltrates consisting of cytotoxic $\mathrm{T}$ cells are present, there is poor response to treatment and poor neurologic outcome. The second group of encephalitides are both paraneoplastic such as anti-NMDAR LE that is associated with teratoma and nonparaneoplastic such as primary autoimmune antiNMDAR LE. They have fewer brain infiltrates, and have significantly better response to treatment with good neurologic outcome, further underscoring the need for timely diagnosis.

Interventions include tumor treatment and immunotherapy (corticosteroids, IVIG or plasmapharesis, rituximab, and cyclophosphamide). If a tumor is found, it should be treated as this could be essential for improvement or stabilization of neurologic symptoms $[12,22]$. Detection of small ovarian teratomas may be impossible, even with CT scans, and some have advocated surgical ovarian removal despite the lack of a radiologically detected tumor.

Most autoimmune encephalitides related to intracellular antigens do not improve but could stabilize following tumor treatment. Tumor removal and treatment with antineoplastic agents has been shown to have a superior outcome over immunotherapy in these cases [23, 24]. Optimal immunotherapy is unknown due to lack of controlled trials There are case reports of patient improvement after corticosteroids and IVIG or plasmapharesis but the effects of these modalities on cytotoxic $\mathrm{T}$ cells are limited. Rituximab has been used but has limited efficacy for paraneoplastic syndromes related to intracellular antigens $[12,25]$. Cyclophosphamide had been effective in some patients $[12,26]$. In summary, identification and eradication of the underlying malignancy is considered 
Table 3. Management Algorithm for Encephalitic Patients in the ICU

History and physical examination

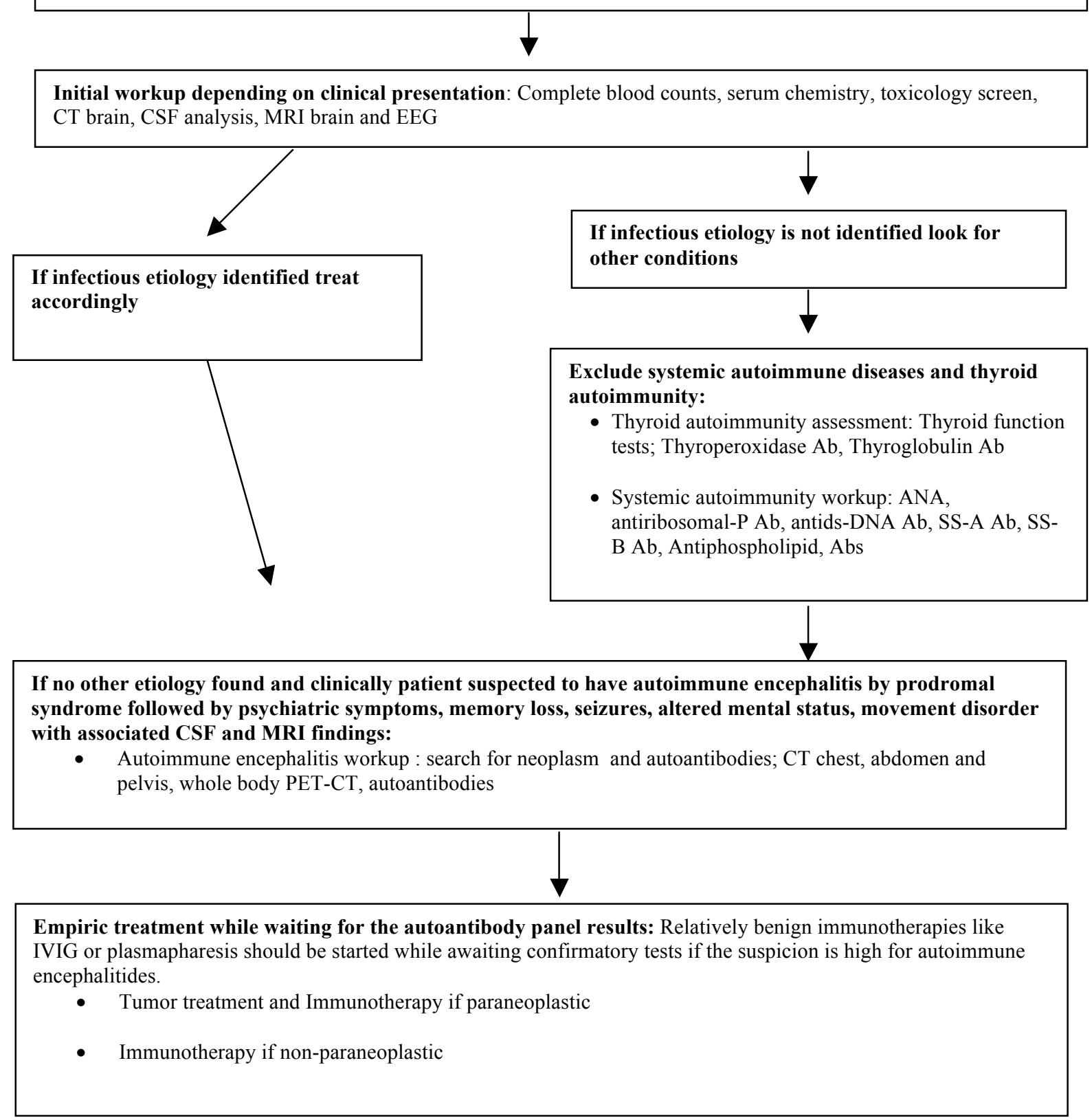

the optimal treatment for paraneoplastic neurological disorders and in patients without active malignancy aggressive immunosuppression can achieve a rate of neurological response similar to those who received chemotherapy for their malignancy [26].

In contrast, autoimmune encephalitides related to cell membrane antigens are significantly more responsive to immunotherapy (corticosteroids, IVIG or plasmapharesis, rituximab, cyclophosphamide). These syndromes may respond to immunotherapy even before the tumor is treated. Once again, tumor removal is essential for more rapid improvement and full recovery $[12,27]$. The condition may relapse if the tumor is not surgically removed [23, 28, 29]. Anti-NMDAR encephalitis has a better prognosis than most other paraneoplastic encephalitides irrespective of the severity of symptoms. Controlled therapeutic trials are lacking but in one case series 9 of 12 patients significantly improved with tumor removal and immunotherapy [7]. 2 of 3 patients who died did not undergo surgery, which emphasizes the importance of tumor removal. 10 out of 11 patients had teratoma and and 1 more patient found to have teratoma at autopsy. Most patients had tumor resection and immunotherapy so relative contribution of these treatments to neurological recovery is difficult to assess in this study.

Our experience suggests corticosteroids should be administered at a dose of $1000 \mathrm{mg}$ of methyl prednisone per day for 3 to 5 days followed by $1 \mathrm{mg}$ per kilogram of maintenance if not contraindicated. For patients who are 
intolerant to steroids or fail to improve with steroids, weekly IVIG at $0.4 \mathrm{~g} / \mathrm{kg}$ for 3-5 days can be attempted. Alternatively, plasma exchange has been reported to be beneficial in some patients. Treatments such as corticosteroids, IVIG and plasmapharesis can be started earlier once the diagnosis is suspected and following the exclusion of other etiologies. It should be kept in mind that tumor detection is often difficult and the results of CSF and serum autoantibodies could take some time since they are performed in highly specialized laboratories. Therapies must be titrated to the clinical response and to the titer of the pathogenic anti-neuronal antibodies in CSF and/or in the serum.

In addition to immunotherapy, critically ill patients will require seizure treatment with standard anti-epileptic drugs. Occasionally, the movement disorders are very difficult to control and patients may require sedative hypnotics, often in very high doses.

\section{CONCLUSION}

Encephalitis is a common diagnosis in the intensive care unit. Following the exclusion of common etiologies and those associated with collagen vascular disease, LE becomes the most common etiology. It should be considered in young women presenting with a subacute onset of psychiatric symptoms without prior psychiatric history. It is accompanied by memory loss, seizures and movement disorder. Prompt recognition of the typical phenotypic presentation of LE should trigger an aggressive search for both autoantibodies and malignancy associated with LE. Tumor removal and immunotherapy is the main mode of treatment. Empiric therapy with corticosteroids, plasmapharesis or IVIG should be considered while awaiting confirmatory laboratory results. Early recognition and treatment often lead to excellent recovery.

\section{CONFLICT OF INTEREST}

The authors confirm that this article content has no conflict of interest.

\section{ACKNOWLEDGEMENTS}

Declared none.

\section{REFERENCES}

[1] Wingfield T, Mchugh C, Vas A, et al. Autoimmune encephalitis: a case series and comprehensive review of the literature. Q J Med 2011; 104: 921-31.

[2] Glaser CA, Honarmand S, Anderson LJ, et al. Beyond viruses: clinical profiles and etiologies associated with encephalitis. Clin Infect Dis 2006; 43: 1565-77.

[3] Gultekin SH, Rosenfeld MR, Voltz R, Eichen J, Posner JB, Dalmau J. Paraneoplastic limbic encephalitis: neurological symptoms, immunological findings and tumor. Brain 2000; 123(Pt 7): 1481-94.

[4] Lawn ND, Westmoreland BF, Kiely MJ. Clinical, magnetic resonance imaging, and electroencephalographic findings in paraneoplastic limbic encephalitis. Mayo Clin Proc 2003; 78: 1363-8.

[5] Daniel SE, Love S, Scaravilli F. Encephalomyeloneuropathy in the absence of a detectable neoplasm: Clinical and postmortem findings in three cases. Acta Neuropathol 1985; 66: 311-7.
[6] Bien CG, Schulze-Bonhage A, Deckert M. Limbic encephalitis not associated with neoplasm as a cause of temporal lobe epilepsy. Neurology 2000; 54: 1823-8.

[7] Dalmau J, Tuzun E, Wu H, et al. Pareneoplastic anti-N-methyl-Daspartate receptor encephalitis associated with ovarian teratoma. Ann Neurol 2007; 61: 25-36.

[8] Pruss H, Dalmau J, Harms L, et al. Retrospective analysis of antiglutamate receptor (type NMDA) antibodies in patients with encephalitis of unknown origin. Neurology 2010; 75: 1735-9.

[9] Corsellis JA, Goldberg GJ, Norton AR. "Limbic encephalitis" and its association with carcinoma. Brain 1968; 91(3): 481-96

[10] Diamond B, Huerta PT, Mina-Osorio P, Kowal C, Volpe BT. Losing your nerves? May be it's the antibodies. Nature Rev 2009; 9: 449-56.

[11] McKeon A, Pittock SJ. Paraneoplastic encephalomyelopathies: pathology and mechanisms. Acta Neuropathol 2011; 122: 381-400.

[12] Tüzün E, Dalmau J. Limbic encephalitis and variants: classification, diagnosis and treatment. Neurologist 2007; 13: 261-71.

[13] Shimazaki H, Ando Y, Nakano I, et al. Reversible limbic encephalitis with antibodies against the membrane of neurons of the hippocampus. J Neurol Neurosurg Psychiat 2007; 78: 324-5.

[14] Irani SR, Vincent A. NMDA receptor antibody encephalitis. Clin Neurol Neurosci Rep 2011; 11: 294-304.

[15] Kleinig T, Thompson PD, Matar W, et al. The distinctive movement disorder of ovarian teratoma-associated encephalitis. Movet Disorders 2008; 23: 1256-61.

[16] Kumei S, Kuzuhara S, Ishihara M, et al. Nationwide survey of acute juvenile female non-herpetic encephalitis in Japan: relationship to antiN-methyl-D-aspartate receptor encephalitis. Intern Med 2009; 48: 6739.

[17] Thieben MJ, Lennon VA, Boeve BF, et al. Potentially reversible autoimmune limbic encephalitis with neuronal potassium channel antibody. Neurology 2004; 62: 1177-82.

[18] Castillo P, Boeve B, Schauble B, et al. Steroid responsive encephalopathy associated with thyroid autoimmunity: clinical and laboratory findings. Neurology 2002; 58: A248 abstract.

[19] Shaw PJ, Walls TJ, Newman PK, et al. Hashimoto's encephalopathy: a steroid responsive disorder associated with hight anti-thyroid antibody titers-report of 5 cases. Neurology 1991; 41: 228-33.

[20] Chong JY, Rowland LP, Utiger RD. Hashimoto encephalopathy: syndrome or myth? Arch Neurol 2003; 60: 164-71.

[21] Leypoldt F, Buchert R, Kleiter I, et al. Fluorodeoxyglucose positron emission tomography in anti-N-methyl-D-aspartate receptor encephalitis: distinct pattern of disease. J Neurosurg Psychiat 2012; 83: 681-66.

[22] Keime-Guibert F, Graus F, Broet P, et al. Clinical outcome of patients with anti-Hu-associated encephalomyelitis after treatment of the tumor. Neurology 1999; 53: 1719-23.

[23] Wingfield T, McHugh C, Vas A, et al. Autoimmune encephalitis: a case series and comprehensive review of the literature. Q J Med 2011; 104: 921-31.

[24] Irani S, Lang B. Autoantibody mediated disorders of the central nervous system. Autoimmunity 2008; 41: 55-6.

[25] Shams'illi S, de Beukelaar J, Gratama JW, et al. An uncontrolled trial of rituximab for antibody associated paraneoplastic neurological syndromes. J Neurol 2006; 16-20.

[26] Vermino S, O’Neill B, Marks R, et al. Immunomodulatory treatment trial for paraneoplastic neurological disorders. Neuro-oncology 2004; 6: 55-62

[27] Lizuka T, Sakai F, Dalmau J. Anti-NMDAR encephalitis in Japan: long term outcome without tumor removal. Neurology 2008; 70(7): 504-11

[28] Dalmau J, Gleichman AJ, Hughes EG, et al. Anti-NMDA-receptor encephalitis: case series and analysis of the effects of antibodies. Lancet Neurol 2008; 7: 1091-8.

[29] Sansing LH, Tuzun E, Ko MW, et al. A patient with encephalitis associated with NMDA receptor antibodies. Nat Clin Pract Neurol 2007; $\quad 67$ : $118-21$ 